\title{
Longterm outcomes after endoscopic mucosal resection for early gastric cancer
}

\author{
Noriya Uedo ${ }^{1}$, Hiroyasu Iishi ${ }^{1}$, Masaharu Tatsuta ${ }^{1}$, Ryu Ishihara ${ }^{1}$, Koji Higashino ${ }^{1}$, Yoji Takeuchi ${ }^{1}$, \\ Kazuho Imanaka ${ }^{1}$, Takuya Yamada ${ }^{1}$, Sachiko Yamamoto ${ }^{1}$, Shunsuke Yamamoto ${ }^{1}$, Hideaki Tsukuma ${ }^{2}$, \\ and SHINGo ISHIGURO ${ }^{3}$ \\ ${ }^{1}$ Department of Gastrointestinal Oncology, Osaka Medical Center for Cancer and Cardiovascular Diseases, 3-3 Nakamichi 1-chome, \\ Higashinari-ku, Osaka 537-8511, Japan \\ ${ }^{2}$ Department of Cancer Control and Statistics, Osaka Medical Center for Cancer and Cardiovascular Diseases, Osaka, Japan \\ ${ }^{3}$ Department of Pathology, Osaka Medical Center for Cancer and Cardiovascular Diseases, Osaka, Japan
}

\begin{abstract}
Background. Despite the widespread use of endoscopic mucosal resection (EMR) in patients with early gastric cancer (EGC), its longterm outcomes have not been fully evaluated. Our aim was to evaluate longterm survival after complete EMR for EGC.

Methods. From patients who underwent EMR between 1978 and 1996 at our center, we enrolled 131 patients with differentiated mucosal EGCs less than $2 \mathrm{~cm}$ (without ulcerative change) that had been completely removed by EMR. The vital status of the patients at the end of December 1998 was confirmed by the hospital cancer registry, which is linked to the Osaka Cancer Registry.

Results. A total of 124 patients $(95 \%)$ were completely followed-up. Two patients $(1.5 \%)$ died of gastric cancer and 26 died of other causes during the mean observation period of 58 months. The overall 5- and 10 -year survival rates were $84 \%$ and $64 \%$, respectively. The disease-specific 5- and 10-year survival rates were $99 \%$ and $99 \%$.

Conclusion. En bloc EMR ensured an excellent prognosis, and should be the first choice of treatment in patients with small differentiated mucosal EGC. Careful histological examination and longterm endoscopic surveillance are important.
\end{abstract}

Key words Early gastric cancer · Endoscopic mucosal resection $\cdot$ Longterm outcomes

\section{Introduction}

Lymph node metastasis is uncommon in early gastric cancers (EGCs), especially in those confined to the

Offprint requests to: N. Uedo

Received: July 4, 2005 / Accepted: December 16, 2005 mucosa $(0-7 \%)$ [1]. Clinicopathological analysis of lymph node-positive mucosal cancers revealed that they were larger and more frequently of undifferentiated type, and that all had ulcerations or scars [2]. Therefore, patients with small differentiated mucosal cancers without ulcerative change have a low risk of lymph node metastasis, and are good candidates for local therapy such as endoscopic mucosal resection (EMR).

In this respect, EMR has been widely performed for EGC in Japan [3] and its indications are delineated in the treatment guidelines of the Japanese Gastric Cancer Association (JGCA) [4]. However, the longterm outcomes of EMR have not been fully evaluated. Because EGC is known to have a long natural history [5], the treatment effect of EMR should be evaluated by longterm follow-up studies.

In this study, we reviewed the prognosis of patients with differentiated mucosal EGC of less than $2 \mathrm{~cm}$ (without ulcerative change), treated by complete EMR.

\section{Patients and methods}

\section{Study subjects}

During the period 1978 to 1996, the clinicopathological data of patients who underwent EMR were stored consecutively in a database that included the location, size, and types of the tumors and the histological findings of the resected specimens, categorized according to the Japanese classification of gastric carcinoma (JCGC) [6]. From the database, we identified 131 patients with 139 EGCs who met our inclusion criteria (Tables 1 and 2 ). These criteria satisfied the indications for EMR in the treatment guidelines issued by the JGCA and curability according to the JCGC $[4,6]$. 
Table 1. Inclusion criteria for the study subjects

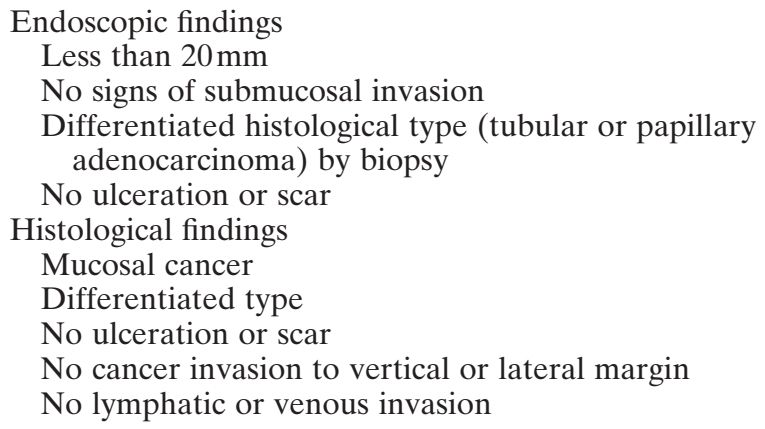

Table 2. Characteristics of the study subjects

\begin{tabular}{lc}
\hline Number of patients & 131 \\
Average age, years $( \pm \mathrm{SD})$ & $66 \pm 9$ \\
Sex & \\
$\quad$ Male & 45 \\
$\quad$ Female & $58 \pm 42$ \\
Mean observation period, months $( \pm \mathrm{SD})$ & $11 \pm 4.6$ \\
Mean tumor size, mm ( \pm SD) & \\
Macroscopic type (No. of tumors, 139) & 31 \\
0-I & 74 \\
0-IIa & 6 \\
0-IIa+IIc & 28 \\
0-IIc & 10 \\
Histological type & \\
Papillary adenocarcinoma & 123 \\
Tubular adenocarcinoma & 6 \\
$\quad$ Well-differentiated & \\
$\quad$ Moderately differentiated &
\end{tabular}

\section{EMR strategies}

EMR was performed by endoscopic snarectomy from 1978 to the middle of 1991 , and subsequently with a submucosal saline injection, using a two-channel endoscope [7].

All resected specimens were retrieved, pinned on hard Styrofoam plates, immersed in formalin, cut parallel into 2-mm serial sections, and subjected to histological examinations for evaluation of the extent of resection and risks of lymph node metastasis.

If lymph node metastasis appeared possible, as evidenced by submucosal invasion, lymphatic or venous invasion, ulcerative change, or positive submucosal margins, the patients were submitted to gastrectomy with lymph node dissection.

Follow-up endoscopy, for detection of local recurrence or metachronous multiple cancers, was scheduled at 1, 3, 6, and 12 months and then yearly after EMR.

\section{Life prognosis investigation}

The life prognoses of the patients were investigated by the Hospital Cancer Registry of Osaka Medical Center for Cancer and Cardiovascular Diseases, which is linked with the Osaka Cancer Registry, a population-based cancer registry covering Osaka Prefecture that has been operating since December 1962 [8]. Prognosis at the end of December 1998 was assessed by referral to residential registrations at municipality offices. Causes of death were confirmed by death certificates. For the outcomes of patients who moved away from Osaka Prefecture, we attempted to obtain details by questionnaires or telephone conversations with their families and referring physicians. Patients with unknown vital status were regarded as censored at the time when they were last known to be alive, and those who underwent gastrectomy were regarded as censored at the time of operation.

The mean estimated 5- and 10-year survival rates of the study subjects were calculated with a cohort survival rate table, provided by the Statistics and Survey Section of the National Cancer Center (http://www.ncc.go.jp/jp/ ncca/cohort01.html).

\section{Informed consent}

Possible risks; complications; anticipated results; and alternative options, such as surgery and no treatment, were explained to all patients, who gave their written informed consent. The study protocol was approved by the Ethics Committee of our institution.

\section{Statistical analysis}

Cumulative overall and disease-specific survivals were estimated using the Kaplan-Meier method. The computer software StatView version 5.0 (SAS Institute, Cary, NC, USA) was used for analysis.

\section{Results}

Vital status was unknown in 6 patients (4.6\%), and 1 patient $(0.76 \%)$ underwent surgical operation because cancer invasion to the muscularis mucosae had raised a suspicion of lymph node metastasis. Thus, 124 patients ( $95 \%)$ were completely followed-up, with a mean observation period of 58 months.

Two patients $(1.5 \%)$ died of gastric cancer (Table 3 ) and 26 died of other causes. One patient, with a $20-\mathrm{mm}$ protruding tumor, who underwent EMR died of gastric cancer 22 months after the EMR. In this patient, review of the stored pathological specimen revealed tumor invasion to the muscularis mucosae and lymphatic invasion despite the database describing the tumor as "completely resected". The other patient, who had a focal cancer in a hyperplastic polyp, showed no sign of local recurrence by surveillance endoscopy for 2 years, 
Table 3. Characteristics of EMR patients who died of gastric cancer

\begin{tabular}{|c|c|c|c|c|c|c|c|c|}
\hline \multirow[b]{2}{*}{$\begin{array}{l}\text { Age } \\
\text { (years) }\end{array}$} & \multirow[b]{2}{*}{$\operatorname{Sex}$} & \multicolumn{6}{|c|}{ Gastric cancer } & \multirow{2}{*}{$\begin{array}{c}\text { Observation } \\
\text { period } \\
\text { (months) }\end{array}$} \\
\hline & & $\begin{array}{l}\text { Size } \\
(\mathrm{mm})\end{array}$ & Type & $\begin{array}{c}\text { Histological } \\
\text { type }\end{array}$ & Depth & $\begin{array}{l}\text { Lymphatic } \\
\text { invasion }\end{array}$ & $\begin{array}{l}\text { Venous } \\
\text { invasion }\end{array}$ & \\
\hline 72 & Female & 20 & 0 -I & pap & $\mathrm{mm}$ & $+^{\mathrm{a}}$ & - & 22 \\
\hline 73 & Female & 15 & $0-\mathrm{I}$ & pap & $\mathrm{m}$ & - & - & 135 \\
\hline
\end{tabular}

pap, papillary adenocarcinoma; $\mathrm{mm}$, muscularis mucosae; $\mathrm{m}$, mucosa

${ }^{a}$ Detected by review of stored pathological specimen
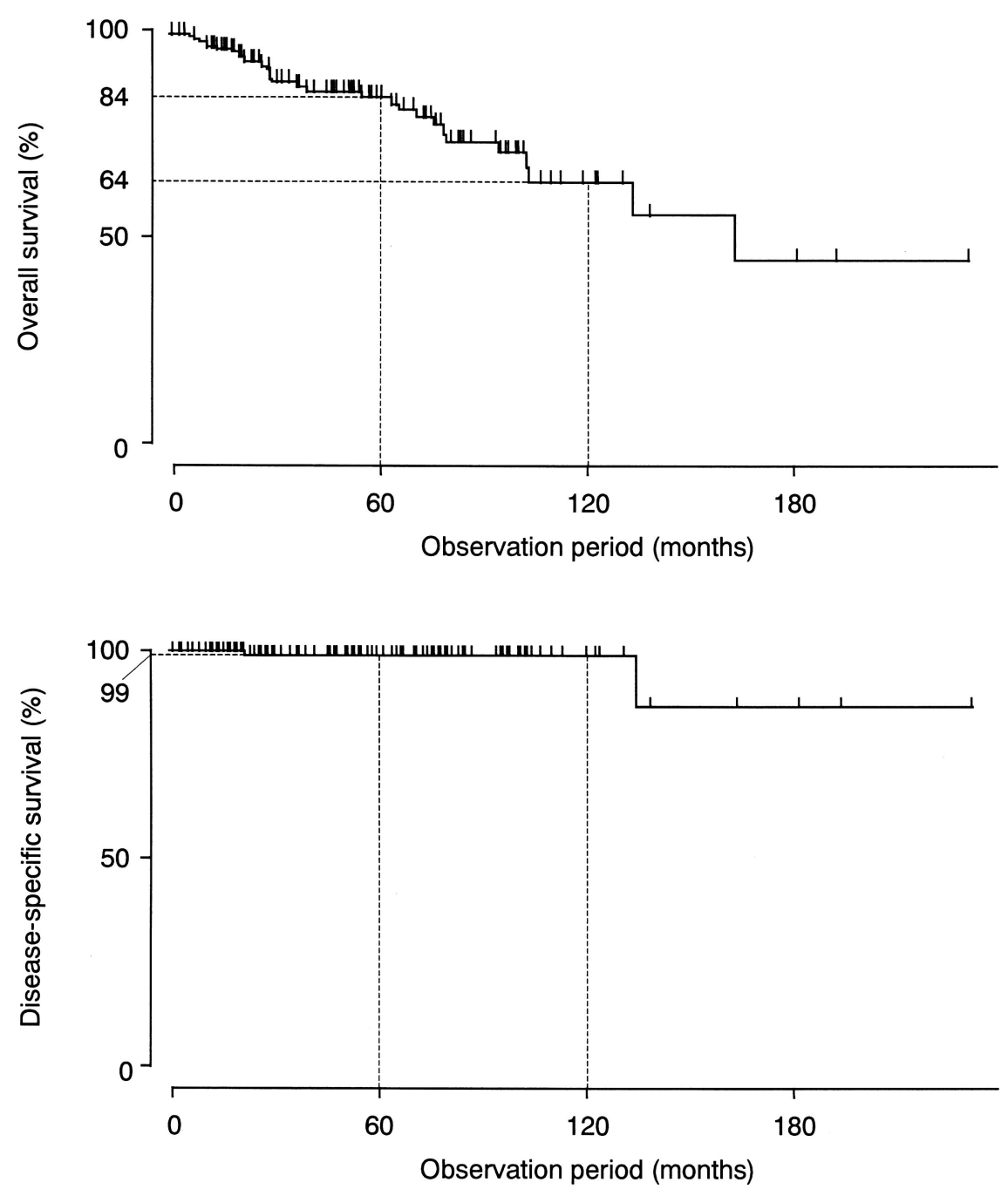

Fig. 1. Kaplan-Meier estimation of overall survival
Fig. 2. Kaplan-Meier estimation of disease-specific survival but then was lost to follow-up. The prognosis data from the Osaka Cancer Registry disclosed her death of gastric cancer 135 months after the EMR.

The overall 5- and 10-year survival rates were $84 \%$ and $64 \%$, respectively (Fig. 1), and they were comparable to the mean estimated 5- and 10-year survival rates of $87 \%$ and $70 \%$. The disease-specific 5- and 10- year survival rates were $99 \%$ and $99 \%$, respectively (Fig. 2).

Seven patients had synchronous double EGCs and one patient had triple EGCs. They were all treated by simultaneous EMR. During the observation period, metachronous multiple EGCs were found in nine patients, who were all treated by EMR. 


\section{Discussion}

This longterm follow-up study indicated that the prognosis of patients with small differentiated-type mucosal EGC (without ulcerative change) treated by EMR was excellent.

Itoh et al. [9] showed that the 5- and 10-year postgastrectomy survival rates for patients with mucosal EGC were $96 \%$ and $92 \%$. The overall survival rates in the current study were relatively low, but the disease-specific survival rates were comparable with those for gastrectomy. Our study subjects included elderly patients and those with complications in major organs, who are not good candidates for surgery. Thus, they may have died of other causes before death ensued from gastric cancer, resulting in overestimation of the disease-specific survival. To correct such bias, the prognosis of patients with EGC who have a low risk of surgery should be evaluated prospectively.

Concerning the good prognosis for $\mathrm{EGC}$, some investigators claim that EGC represents a pseudocancer and rarely affects patients' life prognosis [10]. We have demonstrated that EGC, although it has a long natural history, would progress to an advanced stage and lead to death from gastric cancer [5]. The data derived from that investigation suggested that the 5- and 10-year survival rates of untreated patients with EGC who fulfilled the indications for EMR were $84 \%$ and $30 \%$, respectively. Although those data are not truly comparable with those of the current study, we believe that EMR could reduce gastric cancer deaths in patients with EGC.

The efficacy of endoscopic surveillance should be considered. Multiple gastric cancers have been found in $2 \%-15 \%$ of gastric cancer patients $[11,12]$, and are more frequent in EGC than in advanced cancer [13]. In contrast to gastrectomy, most of the gastric mucosa that may contain areas at high risk for developing EGC such as mucosa with atrophic gastritis and intestinal metaplasia - remain after EMR. Therefore, recognition of multiple cancers is one of the factors that would improve prognosis after EMR. In the present study, nine patients had metachronous multiple cancers, which were all treated by EMR. The patient who died of gastric cancer 135 months after EMR, because of a focal cancer in a hyperplastic polyp, was at no risk of lymph node or distant metastasis. We suspect that she died of a second primary cancer, although we could not obtain the relevant information on the circumstances of her death.

We experienced a patient with EGC who died of gastric cancer 22 months after complete EMR in whom lymphatic invasion was overlooked. Review of the stored pathological specimen revealed papillary adeno- carcinoma invading the muscularis mucosae, with lymphatic invasion, despite the recorded data showing mucosal cancer. This case illustrates the importance of careful histological examination. In fact, among 145 patients who were endoscopically diagnosed as having EGC that fulfilled the indications for EMR and who received en-bloc resection, 13 had submucosal cancer and 1 had mucosal cancer with lymphatic invasion. In differentiated-type submucosal gastric cancer, lymphatic or venous invasion was the most significant determinant of lymph node metastasis, and papillary adenocarcinoma per se had an increased risk of lymph node metastasis [14].

In conclusion, our longterm follow-up study clarified the efficacy of EMR for EGC. En bloc EMR ensured an excellent prognosis, and should be the first choice of treatment in patients with small differentiated mucosal EGC. Careful histological assessment, to determine the risk of lymph node metastasis, and longterm endoscopic surveillance for the detection of multiple cancers are important.

Acknowledgments This study was supported in part by a grant from Daiwa Securities Health Foundation. The authors thank Dr. Massoud Omar for his help and encouragement in the preparation of the manuscript.

\section{References}

1. Everett SM, Axon AT. Early gastric cancer in Europe. Gut 1997:41:142-50.

2. Tsuji N, Ishiguro S, Suzuki N. Risk factors for lymph node metastasis of intramucosal gastric cancer: a case-control study. Gastroenterol Endosc 1999;41:1059-65.

3. Nishi M, Ishihara S, Nakajima T, Ohta K, Ohyama S, Ohta H. Chronological changes of characteristics of early gastric cancer and therapy: experience in the Cancer Institute Hospital of Tokyo, 1950-1994. J Cancer Res Clin Oncol 1995;121:535-41.

4. Nakajima T. Gastric cancer treatment guidelines in Japan. Gastric Cancer 2002;5:1-5.

5. Tsukuma H, Oshima A, Narahara H, Morii T. Natural history of early gastric cancer: a non-concurrent, long term, follow up study. Gut 2000;47:618-21.

6. Japanese Gastric Cancer Association. Japanese classification of gastric carcinoma, 2nd English edition. Gastric Cancer 1998;1: $10-24$.

7. Tada M, Karita M, Yanai H, Takemoto T. Endoscopic therapy of early gastric cancer by strip biopsy. Gan To Kagaku Ryoho (Jpn J Cancer Chemother) 1988;15:1460-5.

8. Parkin DM, Whelan SL, Ferlay J, Teppo L, Thomas DB. editors. Cancer incidence in five continents, vol VIII. IARC Scientific Publications No. 155. Lyon:IARC;2002.

9. Itoh H, Oohata Y, Nakamura K, Nagata T, Mibu R, Nakayama F. Complete 10-year postgastrectomy follow-up of early gastric cancer. Am J Surg 1989;158:14-6.

10. Everett SM, Axon AT. Early gastric cancer: disease or pseudodisease? Lancet 1998;351:1350-2.

11. Brandt D, Muramatsu Y, Ushio K, Mizuguchi Y, Itabashi M, Yoshida S, et al. Synchronous early gastric cancer. Radiology 1989; 173:649-52. 
12. Kodera Y, Yamamura Y, Torii A, Uesaka K, Hirai T, Yasui K, et al. Incidence, diagnosis and significance of multiple gastric cancer. Br J Surg. 1995;82:1540-3.

13. Marrano D, Viti G, Grigioni W, Marra A. Synchronous and metachronous cancer of the stomach. Eur J Surg Oncol 1987; 13: 493-8.
14. Mita T, Shimoda T. Risk factors for lymph node metastasis of submucosal invasive differentiated type gastric carcinoma: clinical significance of histological heterogeneity. J Gastroenterol 2001; 36:661-8. 